\title{
Generalized analytical solution for advection-dispersion equation in finite spatial domain with arbitrary time-dependent inlet boundary condition
}

\author{
J.-S. Chen ${ }^{1}$ and C.-W. Liu ${ }^{2}$ \\ ${ }^{1}$ Graduate Institute of Applied Geology, National Central University, Jhongli City, Taoyuan County 32001, Taiwan (R.O.C.) \\ ${ }^{2}$ Departmnent of Bioenvironmental Systems Engineering, National Taiwan University, Taipei 10617, Taiwan (R.O.C.)
}

Received: 23 March 2011 - Published in Hydrol. Earth Syst. Sci. Discuss.: 26 April 2011

Revised: 8 July 2011 - Accepted: 22 July 2011 - Published: 5 August 2011

\begin{abstract}
This study presents a generalized analytical solution for one-dimensional solute transport in finite spatial domain subject to arbitrary time-dependent inlet boundary condition. The governing equation includes terms accounting for advection, hydrodynamic dispersion, linear equilibrium sorption, and first order decay processes. The generalized analytical solution is derived by using the Laplace transform with respect to time and the generalized integral transform technique with respect to the spatial coordinate. Some special cases are presented and compared to illustrate the robustness of the derived generalized analytical solution. Result shows an excellent agreement between the analytical and numerical solutions. The analytical solutions of the special cases derived in this study have practical applications. Moreover, the derived generalized solution which consists an integral representation is evaluated by the numerical integration to extend its usage. The developed generalized solution offers a convenient tool for further development of analytical solution of specified time-dependent inlet boundary conditions or numerical evaluation of the concentration field for arbitrary time-dependent inlet boundary problem.
\end{abstract}

\section{Introduction}

Solute transport in subsurface is generally described with the advection-dispersion equation (ADE). Analytical solutions for one-, two- and three-dimensional ADEs have been reported in literature for predicting the transport of various contaminants in the semi-finite or infinite spatial domain (e.g., van Genuchten and Alves, 1982; Batu, 1989, 1993,

Correspondence to: J.-S. Chen

(jschen@geo.ncu.edu.tw)
1996; Leij et al., 1991, 1993; Chen et al., 1996; Park and Zhan, 2001; Yeh and Yeh, 2007; Zhan et al., 2009; Chen et al., 2011a). The number of analytical solutions for finite spatial domain is limited compared with semi-finite or infinite spatial domain solutions. The reason for the lack of progress in developing analytical solutions for finite spatial domain is that the solution procedures tend to be relatively cumbersome, requiring complicated or difficult mathematical derivation and manipulations (Peréz Guerrero et al., 2009a, b). In groundwater hydrology, the Laplace transform technique has been widely applied to develop the analytical solutions to ADE. The process of applying Laplace transform to obtain analytical solutions for finite spatial domain in the Laplace space is not complicated, whereas analytically inverting the analytical solution from the Laplace space back to the original time domain is much more difficult. The inverse Laplace transform is mostly performed based on the complex functions and residual theory, thus limiting the numbers and types of the closed-form analytical solutions for finite spatial domain. Accordingly, some researchers used the classic or generalized integral transform technique to develop the analytical solution for solute transport in finite spatial domain. For instance, the analytical solutions for one-dimensional advection-dispersion transport in finite spatial domain subject to first- and third-type inlet boundary conditions were presented by Clearly and Adrian (1973), Selim and Mansell (1976), respectively. van Genuchten and Alves (1982) presented the analytical solution for finite spatial domain associated with exponentially decaying time-dependent inlet boundary condition. Recently, Pérez Guerrero et al. (2009a) presented a general integral transform technique which provides a systematic, efficient, and straightforward approach for deriving the analytical solution of the solute transport within a finite spatial domain. Prior to applying general

Published by Copernicus Publications on behalf of the European Geosciences Union. 
integral transform technique Pérez Guerrero et al. (2009a) suggested that a change-of-variable is carried out to homogenize the inhomogeneous boundary condition using a filter function because that solutions of inhomogeneous problems based on eigenfunction expansions may converge slowly or even exhibit anomalous behavior, especially in the vicinity of the boundaries as noted by Ozisik (1980) and Cotta and Mikhailov (1997). For the case of the transport in a finite spatial domain associated with time-invariant boundary conditions, the filter function for homogenizing the inhomogeneous boundary condition can be easily derived. However, the procedure for obtaining the filter function for finite spatial domain with time-dependent boundary condition is much more complicated because of the need to define the filter function over both the time and spatial domain. Accordingly, the application of generalized integral technique to obtain the analytical solution for ADE in finite spatial domain is limited to time-invariant constant and time-dependent exponentially decaying inlet boundary condition (Pérez Guerrero et al., 2009a, b, 2010a; Pérez Guerrero and Skaggs, 2010b).

As the authors aware, analytical solution for finite spatial domain associated with arbitrary time-dependent inlet boundary condition has not been reported in literature yet. In many instances the solute transport problems may involve the various types of time-dependent inlet boundary conditions. For example, such as naturally occurring isotopes moving from a flow-through lake into an aquifer can be dependent upon natural, cyclic, water-quality variations; or liquid waste disposal of a human-made system operating on a periodic cycle. Additionally, the tracer test may be performed by adopting a time-dependent input mode. The solution for arbitrary time-dependent input function should be useful for describing solute transport in a natural or human-made system in which the input at a boundary is a function of time (Logan and Zlotnik, 1995, 1996). In the present study we attempt to derive the generalized analytical solution for ADE in finite spatial domain subject to arbitrary time-dependent inlet boundary condition. Laplace transform in combination with generalized integral transform is used to obtain the generalized analytical solution. Laplace transform is applied to convert the time-dependent inhomogeneous boundary condition into non-time-dependent boundary condition. Thus, the constraint in obtaining the filter function for transport in finite spatial domain with transient boundary condition can be overcome. The generalized analytical solution is applied to derive some specific analytical solutions to demonstrate its practical applications. Moreover, the generalized analytical solution which consists of a definite integral expression is evaluated by means of numerical integration technique to extend its applicability for describing solute transport associated with arbitrary time-dependent inlet boundary condition.

\section{Governing equations}

Herein we consider a problem of one-dimensional advectivedispersive solute transport in finite spatial domain subject to arbitrary time-dependent inlet boundary condition. The solute transport equation incorporates terms accounting for advection, dispersion, linear equilibrium sorption, and firstorder decay processes. The governing equation for this solute transport problem is expressed as

$D \frac{\partial^{2} C}{\partial x^{2}}-V \frac{\partial C}{\partial x}-k C=R \frac{\partial C}{\partial t}$

where $C(x, t)$ is the solute concentration; $x$ is the spatial coordinate; $t$ is time; $V=\frac{U}{\phi}$ stands for the average linear velocity of the pore fluid, where $U$ is specific discharge, or Darcian velocity and $\phi$ is porosity; $D$ represents the longitudinal dispersion coefficient; $R$ is the retardation coefficient of the solute, and $k$ is the first-order decay rate constant.

The initial and boundary conditions considered herein are

$$
\begin{aligned}
& C(x, t=0)=0 \quad 0 \leq x \leq L \\
& V C(x=0, t)-D \frac{\partial C(x=0, t)}{\partial x}=V f(t) t>0 \\
& \frac{\partial C(x=L, y, t)}{\partial x}=0 t>0
\end{aligned}
$$

where $L$ is the length of the finite spatial domain, $f(t)$ represents the arbitrary solute concentration applied at $x=0$ which will be specified later.

Inserting the following dimensionless variables, $x_{\mathrm{D}}=x / L$ and $t_{\mathrm{D}}=V t / \mathrm{RL}$ into Eqs. (1) to (4) yields the following governing equation and its auxiliary initial and boundary conditions in dimensionless form as

$$
\begin{aligned}
& \frac{1}{\operatorname{Pe}} \frac{\partial^{2} C}{\partial x_{\mathrm{D}}^{2}}-\frac{\partial C}{\partial x_{\mathrm{D}}}-k_{\mathrm{D}} C=\frac{\partial C}{\partial t_{\mathrm{D}}} \\
& C\left(x_{\mathrm{D}}, t_{\mathrm{D}}=0\right)=0 \quad 0 \leq x_{\mathrm{D}} \leq 1 \\
& C\left(x=0, t_{\mathrm{D}}\right)-\frac{1}{\operatorname{Pe}} \frac{\partial C\left(x_{\mathrm{D}}=0, t_{\mathrm{D}}\right)}{\partial x_{\mathrm{D}}}=f\left(\frac{\mathrm{RL}}{V} t_{\mathrm{D}}\right) t_{\mathrm{D}}>0 \\
& \frac{\partial C\left(x_{\mathrm{D}}=1, t_{\mathrm{D}}\right)}{\partial x_{\mathrm{D}}}=0 t_{\mathrm{D}}>0
\end{aligned}
$$

where $\mathrm{Pe}=\mathrm{VL} / D$ and $k_{\mathrm{D}}=k L / V$.

\section{Derivation of the Generalized Analytical Solution}

The analytical solution to Eq. (5) subject to Eqs. (6) to (8) is derived using the Laplace transform with respect to $t_{\mathrm{D}}$ and the general integral transform technique with respect to $x_{\mathrm{D}}$.

First, the Laplace transform is carried out on Eq. (5) with the help of Eq. (6) and its auxiliary boundary conditions in 
Eqs. (7) and (8) with respect to $t_{\mathrm{D}}$. After the Laplace transform procedure the governing equation (Eq. 5) and boundary conditions (Eqs. 7 to 8) become

$\frac{1}{\mathrm{Pe}} \frac{d^{2} C_{\mathrm{L}}}{d x_{\mathrm{D}}^{2}}-\frac{d C_{\mathrm{L}}}{d x_{\mathrm{D}}}-\left(k_{\mathrm{D}}+s\right) C_{\mathrm{L}}=0$

$C_{\mathrm{L}}\left(x_{\mathrm{D}}=0, s\right)-\frac{1}{\mathrm{Pe}} \frac{\partial C_{\mathrm{L}}\left(x_{\mathrm{D}}=0, s\right)}{\partial x_{\mathrm{D}}}=f_{\mathrm{L}}(s)$

$\frac{d C_{\mathrm{L}}\left(x_{\mathrm{D}}=1, s\right)}{d x_{\mathrm{D}}}=0$

where $s$ denotes the dimensionless Laplace transform parameter and $C_{\mathrm{L}}\left(x_{\mathrm{D}}, s\right)$, and $f_{\mathrm{L}}(s)$ represent the Laplace transforms of $C\left(x_{\mathrm{D}}, t_{\mathrm{D}}\right)$ and $f\left(\mathrm{RL} t_{\mathrm{D}} / V\right)$, respectively, which are defined by the following equations

$C_{\mathrm{L}}\left(x_{\mathrm{D}}, s\right)=L\left[C\left(x_{\mathrm{D}}, t_{\mathrm{D}}\right)\right]=\int_{0}^{\infty} C\left(x_{\mathrm{D}}, t_{\mathrm{D}}\right) e^{-s t_{\mathrm{D}}} d t_{\mathrm{D}}$

$f_{\mathrm{L}}(s)=L\left[f\left(\frac{\mathrm{RL}}{V} t_{\mathrm{D}}\right)\right]=\int_{0}^{\infty} f\left(\frac{\mathrm{RL}}{V} t_{\mathrm{D}}\right) e^{-s t_{\mathrm{D}}} d t_{\mathrm{D}}$

The general integral transform technique is then adopted to analytically solve the Eq. (9) and its auxiliary boundary conditions in Eq. (10). Further information regarding the use of the generalized integral transform can be found in Pérez Guerreo et al. (2009a, b, 2010a; Chen et al., 2011b). Prior to applying the general integral transform technique a change-of-variable is carried out to homogenize the boundary condition in Eq. (10) and to covert Eq. (9) into a purely diffusive type differential equation. This approach was demonstrated previously by Peréz Guerreo et al. (2009a). Inserting the variable change $C_{\mathrm{V}}\left(x_{\mathrm{D}}, s\right)=$ $\left[C_{\mathrm{L}}\left(x_{\mathrm{D}}, s\right)-f_{\mathrm{L}}(s)\right] \exp \left(-\frac{\mathrm{Pe}}{2} x_{\mathrm{D}}\right)$, Eqs. (9)-(11) can be written in terms of $C_{\mathrm{V}}\left(x_{\mathrm{D}}, s\right)$ as

$$
\begin{aligned}
& \frac{1}{\mathrm{Pe}} \frac{d^{2} C_{\mathrm{V}}}{d x_{\mathrm{D}}^{2}}-\left(\frac{\mathrm{Pe}}{4}+k_{\mathrm{D}}+s\right) C_{\mathrm{V}}=\exp \left(-\frac{\mathrm{Pe}}{2} x_{\mathrm{D}}\right)\left(k_{\mathrm{D}}+s\right) f_{\mathrm{L}}(s) \\
& \frac{d C_{\mathrm{V}}\left(x_{\mathrm{D}}=0, s\right)}{d x_{\mathrm{D}}}-\frac{\mathrm{Pe}}{2} C_{\mathrm{V}}\left(x_{\mathrm{D}}=0, s\right)=0 \\
& \frac{d C_{\mathrm{V}}\left(x_{\mathrm{D}}=1, s\right)}{d x_{\mathrm{D}}}+\frac{\mathrm{Pe}}{2} C_{\mathrm{V}}\left(x_{\mathrm{D}}=1, s\right)=0
\end{aligned}
$$

Following the procedures of the generalized integral transform, the eigenfunction is determined from the following Sturm-Liouville problem with the same kinds of boundary conditions as specified for $C_{\mathrm{V}}\left(x_{\mathrm{D}}, s\right)$ :

$$
\begin{aligned}
& \frac{d^{2} K\left(x_{\mathrm{D}}\right)}{d x_{\mathrm{D}}^{2}}+\psi^{2} K\left(x_{\mathrm{D}}\right)=0 \\
& \frac{d K\left(x_{\mathrm{D}}=0\right)}{d x_{\mathrm{D}}}-\frac{\mathrm{Pe}}{2} K\left(x_{\mathrm{D}}=0\right)=0
\end{aligned}
$$

$$
\frac{d K\left(x_{\mathrm{D}}=1\right)}{d x_{\mathrm{D}}}+\frac{\mathrm{Pe}}{2} K\left(x_{\mathrm{D}}=1\right)=0
$$

Solving for Eqs. (17)-(19), we have the following normalized eigenfunction

$$
K\left(\psi_{m}, z_{\mathrm{D}}\right)=\frac{\sqrt{2}\left[\psi_{m} \cos \left(\psi_{m} z_{\mathrm{D}}\right)+\frac{\mathrm{Pe}}{2} \sin \left(\psi_{m} z_{\mathrm{D}}\right)\right]}{\left(\frac{P e^{2}}{4}+\frac{\mathrm{Pe}}{2}+\psi_{m}^{2}\right)^{\frac{1}{2}}}
$$

where $\psi_{m}$ is the eigenvalue determined from the following equation:

$\psi_{m} \cot \psi_{m}-\frac{\psi_{m}^{2}}{\mathrm{Pe}}+\frac{\mathrm{Pe}}{4}=0$

The generalized integral transform pairs are readily defined as

$$
\begin{aligned}
& \overline{C_{\mathrm{V}}}\left(\psi_{m}, s\right)=\int_{0}^{1} K\left(\psi_{m}, x_{\mathrm{D}}\right) C_{\mathrm{V}}\left(x_{\mathrm{D}}, s\right) d x_{\mathrm{D}} \\
& C_{\mathrm{V}}\left(x_{\mathrm{D}}, t_{\mathrm{D}}\right)=\sum_{m=1}^{\infty} K\left(\psi_{m}, x_{\mathrm{D}}\right) C_{\mathrm{V}}\left(\psi_{m}, s\right)
\end{aligned}
$$

Making use of the above generalized integral transform on Eq. (14) and solving for $\overline{C_{\mathrm{V}}}\left(x_{\mathrm{D}}, s\right)$, one obtain

$$
\begin{aligned}
& \overline{C_{\mathrm{V}}}\left(x_{\mathrm{D}}, s\right)= \\
& \frac{-\sqrt{2} \psi_{m} \mathrm{Pe}}{\left(\frac{\mathrm{Pe}^{2}}{4}+\frac{\mathrm{Pe}}{2}+\psi_{m}^{2}\right)^{\frac{1}{2}}\left(\frac{\mathrm{Pe}^{2}}{4}+\psi_{m}^{2}\right)} \cdot \frac{s+k_{\mathrm{D}}}{s+\frac{\psi_{m}^{2}}{\mathrm{Pe}}+\frac{\mathrm{Pe}}{4}+k_{\mathrm{D}}+s} f_{\mathrm{L}}(s)
\end{aligned}
$$

The analytical solution in original domain can readily be obtained by successively applications of the general integral transform inversion (Eq. 23), change of variable, as well as the Laplace transform inversion. The inverse Laplace transform is achieved using convolution theorem. Following the aforementioned procedures, the final analytical solution can be expressed in dimensionless form as

$C\left(x_{\mathrm{D}}, t_{\mathrm{D}}\right)=f\left(\frac{\mathrm{RL}}{V} t_{\mathrm{D}}\right)-\sum_{m=1}^{\infty} \exp \left(\frac{\mathrm{Pe}}{2} x_{\mathrm{D}}\right) E\left(\psi_{m}, x_{\mathrm{D}}\right) F\left(t_{\mathrm{D}}\right)$

where $E\left(\psi_{m}, x_{\mathrm{D}}\right)=\frac{2 P e \psi_{m}\left[\psi_{m} \cos \left(\psi_{m} x_{\mathrm{D}}\right)+\frac{\mathrm{Pe}}{2} \sin \left(\psi_{m} x_{\mathrm{D}}\right)\right]}{\left(\frac{P e^{2}}{4}+\frac{\mathrm{Pe}}{2}+\psi_{m}^{2}\right)\left(\frac{P e^{2}}{4}+\psi_{m}^{2}\right)}$,

$F\left(t_{\mathrm{D}}\right)=f\left(\frac{\mathrm{RL}}{V} t_{\mathrm{D}}\right)-\left(\frac{\psi_{m}^{2}}{\mathrm{Pe}}+\frac{\mathrm{Pe}}{4}\right) e^{-\left(\frac{\psi_{m}^{2}}{\mathrm{Pe}}+\frac{\mathrm{Pe}}{4}+k_{\mathrm{D}}\right) t_{\mathrm{D}}}$

$\int_{0}^{t_{\mathrm{D}}} f\left(\frac{\mathrm{RL}}{V} \tau\right) e^{\left(\frac{\psi_{m}^{2}}{\mathrm{Pe}}+\frac{\mathrm{Pe}}{4}+k_{\mathrm{D}}\right) \tau} d \tau$ 
Table 1. Three time-dependent input functions and their corresponding analytical solutions. The solutions for constant and exponential decaying time-dependent input functions are the same as those reported in literature.

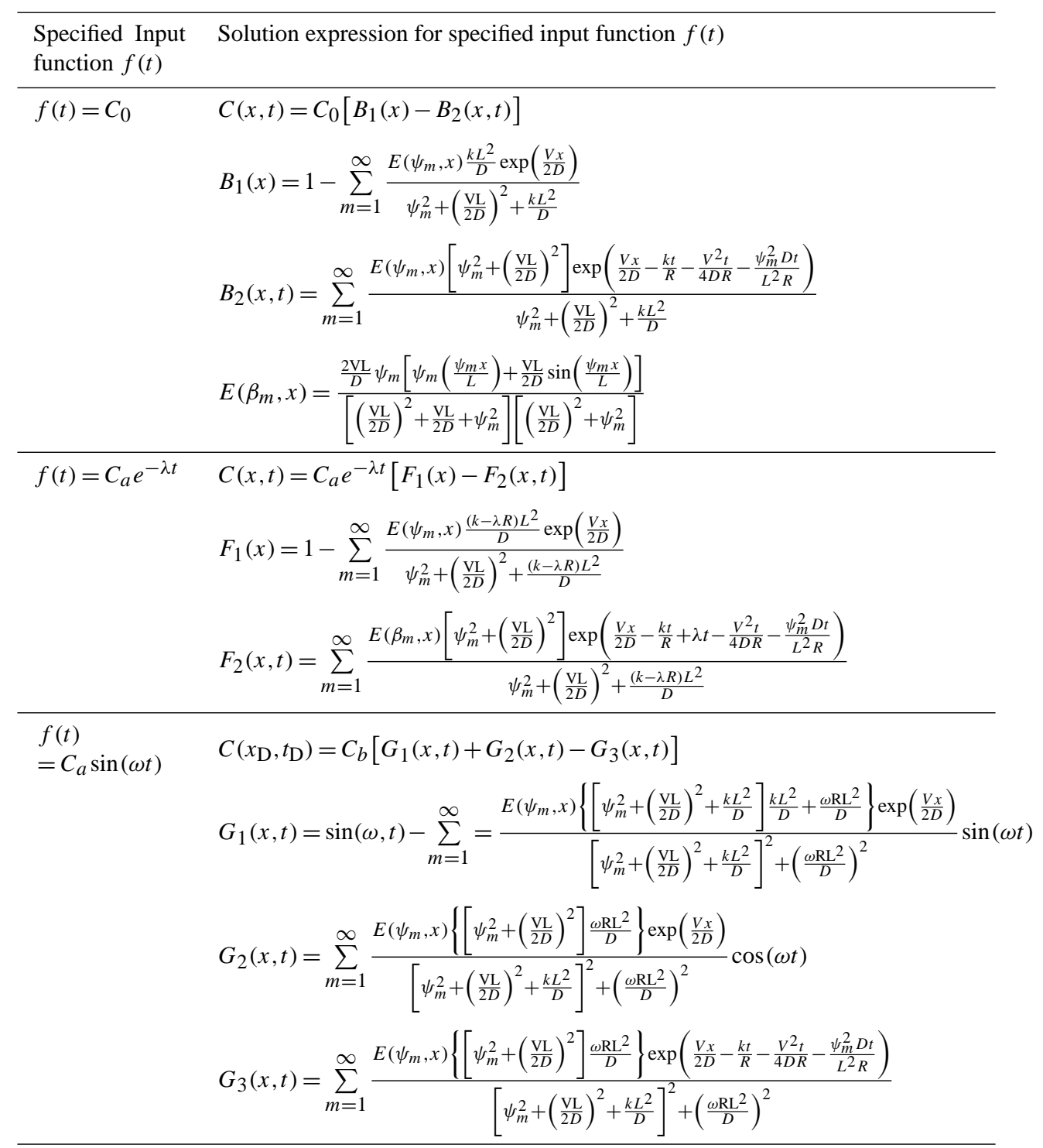

\section{Results and discussion}

\subsection{Development of specific solutions using the generalized analytical solution}

The generalized analytical solution (Eq. 24) provides useful foundation for deriving specific analytical solutions having practical applications. Solution for specified timedependent input function can be readily derived by substituting $f\left(\mathrm{RL} t_{\mathrm{D}} / V\right)$ into the integral expression of Eq. (24). In this study three specific analytical solutions for constant, exponentially decaying and sinusoidally periodic timedependent input functions are derived using integral expres- sion of Eq. (24) (Detailed derivation is provided in Appendix). Table 1 summarizes three specified time-dependent input functions and their corresponding analytical solutions. The solutions for constant and exponentially decaying input functions have previously presented in literature (van Genuchten and Alves, 1982). The solutions for constant and exponentially decaying time-dependent input functions in Table 1 are the same as those reported in literature.

The solution for a finite spatial domain associated with sinusoidally periodic boundary condition with has not been presented in literature. The specific analytical solution for sinusoidally periodic input function is in the form of the sum of the infinite series expansion and can be straightforwardly 
Table 2. Descriptive simulation conditions and transport parameters.

\begin{tabular}{lc}
\hline Parameter & Value \\
\hline $\begin{array}{l}\text { Domain length } \\
L[\mathrm{~m}]\end{array}$ & 100 \\
$\begin{array}{l}\text { Average velocity } \\
V\left[\mathrm{~m} \mathrm{day}^{-1}\right]\end{array}$ & 1 \\
Longitudinal dispersion coefficient $^{2}$ & \\
$D$ [ $^{2}$ day $\left.^{-1}\right]$ & 20 \\
Retardation factor $\left.{ }^{-1}\right]$ & \\
$\begin{array}{l}\text { First decay rate constant } \\
k\left[1 \text { day }^{-1}\right]\end{array}$ & 1 \\
$\begin{array}{l}\text { Frequency of sinusoidal } \\
\text { periodic input function }\end{array}$ & 0.002 \\
$\omega\left[1\right.$ day $\left.^{-1}\right]$ & \\
$\begin{array}{l}\text { Decay rate constant of exponential } \\
\text { decaying input function }\end{array}$ & \\
$\lambda\left[1\right.$ day $\left.^{-1}\right]$ & 0.01 \\
\hline
\end{tabular}

evaluated. Generally, the number of the terms in the infinite series expansion plays a key role in determining the accurate result. Accordingly, we are interested to examine how many terms are required to numerically determine the accurate solution. The parameter values for the numerical results for sinusoidal periodic input function are summarized in Table 2. Table 3 illustrates the convergence of the numerical evaluation of analytical solution for the sinusoidally periodic input. The required number of terms drastically increases with increasing Pe. Numbers of terms 10, 60 and 1800 can achieve convergence to 4 decimal places for Pe equal to 1 , 10 and 50. After determining the number of terms for solution convergence we compare the developed periodic analytical solution with the corresponding numerical solution to examine the correctness of the mathematical derivations and manipulations in the solution development for sinusoidal periodic input function. The numerical solution is generated using the Laplace transform finite difference (LTFD) technique proposed by Moridis and Reddel (1991). The LTFD technique has several advantages over the classical finite difference method. The input parameter values are the same as those in Table 2. Figure 1 depicts the breakthrough curves observed at $x=100 \mathrm{~m}$ obtained from the specific analytical solution and the corresponding numerical solution. As expected, the developed analytical solution agrees well with the corresponding numerical solution.

\subsection{Effects of $D, k, V$ on periodic solute transport}

After validating the analytical solution for sinusoidally periodic input function, we use this analytical solution to investigate the effect of longitudinal dispersion coefficient $(D)$,

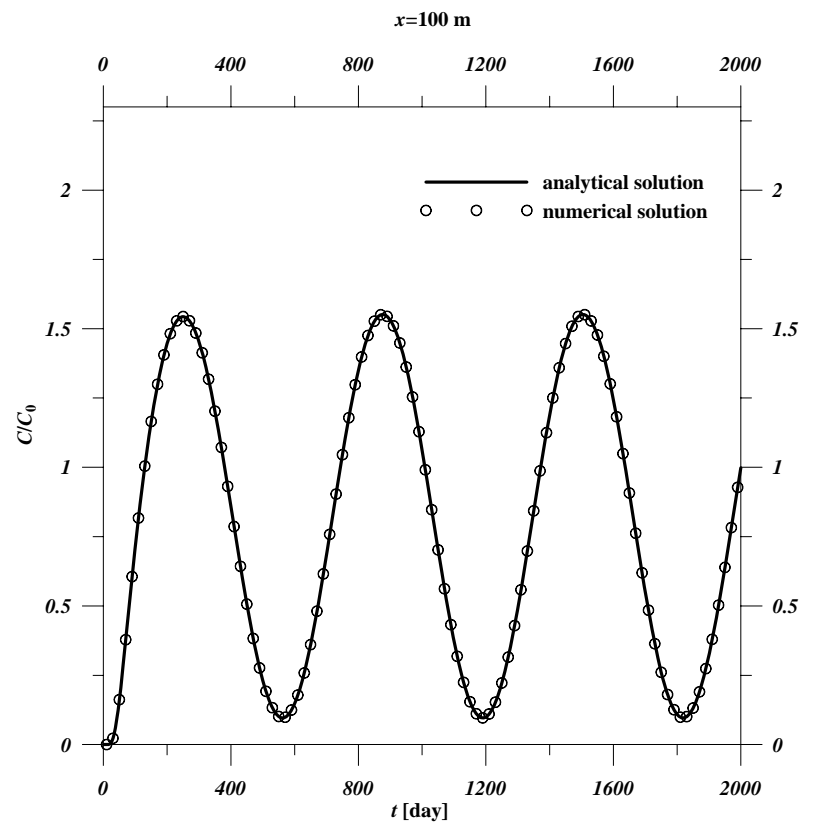

Fig. 1. Comparison of the breakthrough curves at $x=100 \mathrm{~m}$ obtained from the developed specific analytical solution for sinusoidally periodic input function $(f(t)=1+\sin t)$ and the corresponding numerical solution.

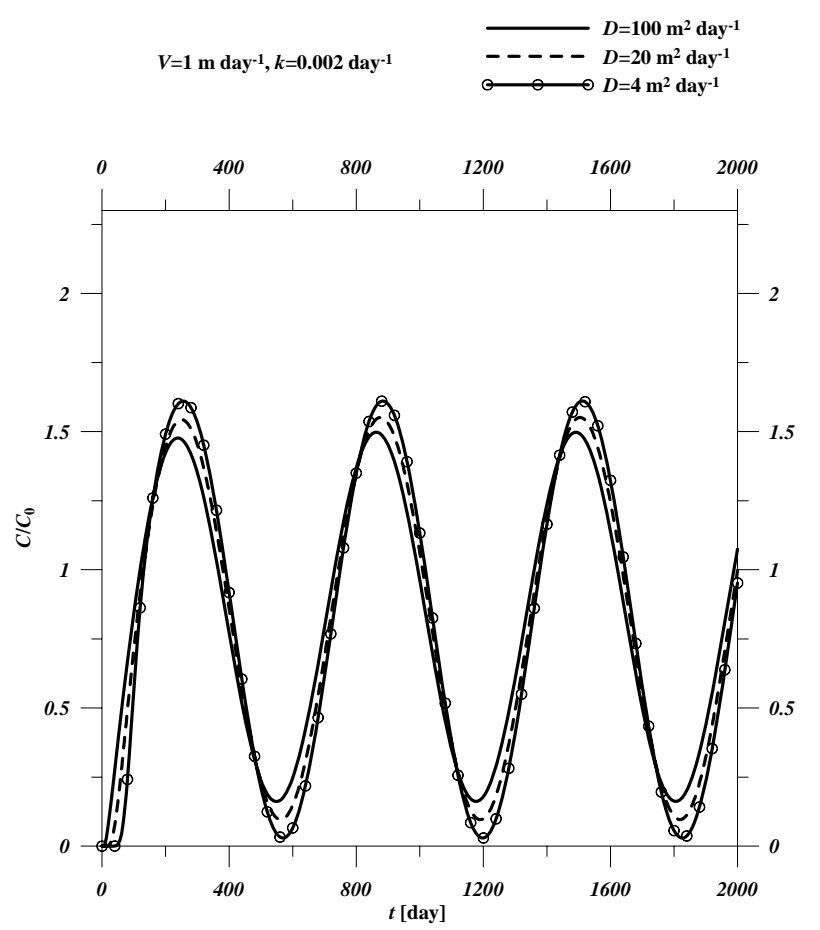

Fig. 2. Comparison of the breakthrough curves at $x=100 \mathrm{~m}$ for different $D$. The sinusoidal periodic input function is $f(t)=1+$ $\sin t$. Parameter $D$ is varied and other parameters are kept constant. 
Table 3. Solution convergence for sinusoidal periodic function $(1+$ $\sin t)(M$ is number of terms summed).

\begin{tabular}{rccccc}
\hline \multicolumn{5}{c}{$P e=1$} \\
\hline [day] & $M=2$ & $M=4$ & $M=6$ & $M=8$ & $M=10$ \\
\hline 16 & 0.2798 & 0.2781 & 0.2780 & 0.2779 & 0.2779 \\
32 & 0.6697 & 0.6681 & 0.6681 & 0.6681 & 0.6681 \\
48 & 1.0033 & 1.0022 & 1.0021 & 1.0021 & 1.0021 \\
64 & 1.2614 & 1.2609 & 1.2608 & 1.2608 & 1.2608 \\
80 & 1.4237 & 1.4237 & 1.4237 & 1.4237 & 1.4237 \\
96 & 1.4762 & 1.4767 & 1.4768 & 1.4768 & 1.4768 \\
112 & 1.4174 & 1.4183 & 1.4184 & 1.4184 & 1.4184 \\
128 & 1.2605 & 1.2616 & 1.2617 & 1.2617 & 1.2617 \\
144 & 1.0325 & 1.0336 & 1.0337 & 1.0337 & 1.0337 \\
160 & 0.7708 & 0.7716 & 0.7717 & 0.7717 & 0.7717 \\
176 & 0.5173 & 0.5178 & 0.5178 & 0.5178 & 0.5178 \\
192 & 0.3127 & 0.3126 & 0.3126 & 0.3126 & 0.3126 \\
208 & 0.1893 & 0.1887 & 0.1886 & 0.1886 & 0.1886 \\
224 & 0.1669 & 0.1658 & 0.1657 & 0.1657 & 0.1657 \\
140 & 0.2491 & 0.2475 & 0.2475 & 0.2474 & 0.2474 \\
256 & 0.4229 & 0.4212 & 0.4211 & 0.4211 & 0.4211 \\
272 & 0.6609 & 0.6592 & 0.6591 & 0.6591 & 0.6591 \\
288 & 0.9256 & 0.9242 & 0.9241 & 0.9241 & 0.9241 \\
304 & 1.1752 & 1.1742 & 1.1742 & 1.1742 & 1.1742 \\
320 & 1.3702 & 1.3698 & 1.3698 & 1.3698 & 1.3698 \\
\hline & \multicolumn{5}{c}{$P e=5$} \\
\hline [day] & $M=10$ & $M=15$ & $M=20$ & $M=25$ & $M=30$ \\
16 & 0.0771 & 0.0766 & 0.0767 & 0.0766 & 0.0766 \\
32 & 0.4920 & 0.4916 & 0.4916 & 0.4916 & 0.4916 \\
48 & 0.9130 & 0.9127 & 0.9128 & 0.9127 & 0.9127 \\
64 & 1.2352 & 1.2350 & 1.2351 & 1.2351 & 1.2351 \\
80 & 1.4468 & 1.4468 & 1.4468 & 1.4468 & 1.4468 \\
96 & 1.5393 & 1.5394 & 1.5394 & 1.5394 & 1.5394 \\
112 & 1.5098 & 1.5101 & 1.5101 & 1.5101 & 1.5101 \\
128 & 1.3683 & 1.3686 & 1.3685 & 1.3685 & 1.3685 \\
144 & 1.1391 & 1.1394 & 1.1394 & 1.1394 & 1.1394 \\
160 & 0.8595 & 0.8597 & 0.8597 & 0.8597 & 0.8597 \\
176 & 0.5739 & 0.5740 & 0.5740 & 0.5740 & 0.5740 \\
192 & 0.3276 & 0.3276 & 0.3276 & 0.3276 & 0.3276 \\
208 & 0.1597 & 0.1595 & 0.1595 & 0.1595 & 0.1595 \\
224 & 0.0965 & 0.0962 & 0.0963 & 0.0962 & 0.0962 \\
140 & 0.1482 & 0.1477 & 0.1478 & 0.1478 & 0.1478 \\
256 & 0.3065 & 0.3060 & 0.3061 & 0.3061 & 0.3061 \\
272 & 0.5465 & 0.5460 & 0.5461 & 0.5461 & 0.5461 \\
288 & 0.8302 & 0.8298 & 0.8299 & 0.8299 & 0.8299 \\
304 & 1.1130 & 1.1127 & 1.1128 & 1.1127 & 1.1127 \\
320 & 1.3500 & 1.3499 & 1.3500 & 1.3499 & 1.3499 \\
\hline & & & & &
\end{tabular}

first-order decay constant $(k)$, and average linear velocity of the pore fluid $(V)$ on the periodic solute transport. Each of the three parameters, namely $D, k$, and $V$ is parametrically varied respectively, while the other parameters are kept constant. It is observed in Fig. 2 that increasing $D$ will decrease the amplitude of the periodic concentration wave due to larger spreading of the solute mass. In Fig. 3, a lower concentration is observed at the crest and trough of concentration wave for larger $k$ due to the decay effect. Examination of Fig. 4 clearly shows that the smaller amplitude of the periodic concentration wave and lower the concentration at the crest and trough of the concentration wave for lower $V$ be-
Table 3. Continued.

\begin{tabular}{rrrrrr}
\hline \multicolumn{5}{c}{$P e=25$} \\
\hline$t$ [day] & $M=200$ & $M=400$ & $M=600$ & $M=800$ & $M=1000$ \\
16 & 0.0017 & 0.0005 & 0.0005 & 0.0004 & 0.0004 \\
32 & 0.2427 & 0.2417 & 0.2416 & 0.2416 & 0.2416 \\
48 & 0.8634 & 0.8627 & 0.8626 & 0.8626 & 0.8626 \\
64 & 1.2601 & 1.2598 & 1.2597 & 1.2597 & 1.2597 \\
80 & 1.4908 & 1.4909 & 1.4909 & 1.4909 & 1.4909 \\
96 & 1.6008 & 1.6012 & 1.6012 & 1.6012 & 1.6012 \\
112 & 1.5859 & 1.5866 & 1.5866 & 1.5866 & 1.5866 \\
128 & 1.4500 & 1.4508 & 1.4508 & 1.4508 & 1.4508 \\
144 & 1.2146 & 1.2154 & 1.2155 & 1.2155 & 1.2155 \\
160 & 0.9170 & 0.9176 & 0.9176 & 0.9177 & 0.9177 \\
176 & 0.6041 & 0.6044 & 0.6044 & 0.6044 & 0.6044 \\
192 & 0.3253 & 0.3252 & 0.3252 & 0.3252 & 0.3252 \\
208 & 0.1247 & 0.1242 & 0.1241 & 0.1241 & 0.1241 \\
224 & 0.0338 & 0.0329 & 0.0329 & 0.0329 & 0.0329 \\
140 & 0.0671 & 0.0660 & 0.0659 & 0.0659 & 0.0659 \\
256 & 0.2193 & 0.2181 & 0.2180 & 0.2180 & 0.2180 \\
272 & 0.4664 & 0.4652 & 0.4651 & 0.4651 & 0.4651 \\
288 & 0.7693 & 0.7683 & 0.7683 & 0.7683 & 0.7682 \\
304 & 1.0803 & 1.0796 & 1.0796 & 1.0796 & 1.0796 \\
320 & 1.3502 & 1.3499 & 1.3499 & 1.3499 & 1.3499 \\
\hline
\end{tabular}

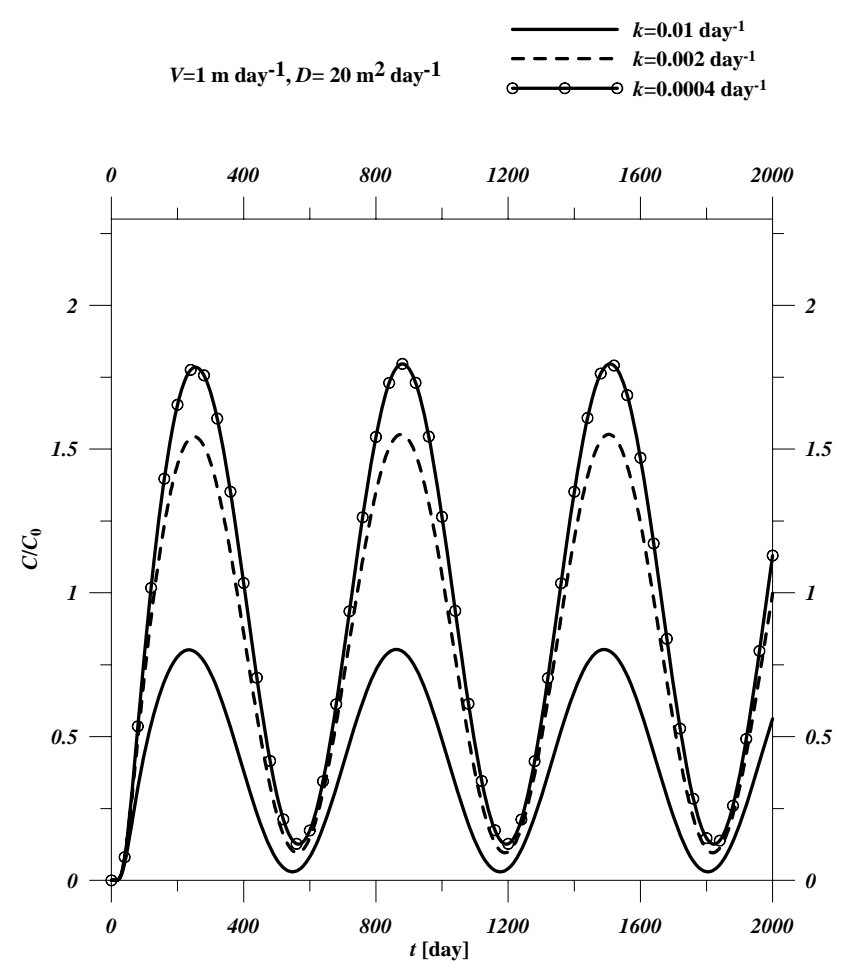

Fig. 3. Comparison of the breakthrough curves at $x=100 \mathrm{~m}$ for different $k$. The sinusoidally periodic input function is $f(t)=1+$ $\sin t$. Parameter $k$ is varied and other parameters are kept constant.

cause the spreading effect by dispersion processes and concentration reduction effect by decay process is enhanced for long solute resident time under low $V$ condition. 


\subsection{Evaluation of the generalized analytical solution using numerical integration}

In Sect. 4.1 we derive some specific analytical solutions using the developed generalized analytical solution (Eq. 24) by substituting the specified time-dependent input function into the integral expression. However, in many instances the development of the specific analytical solution is difficult or prohibited, therefore, the numerical integration method need to be used to evaluate the result of Eq. (24). The reason for using numerical integration method may be that the antiderivative for the specified input function is impossible, or difficult to find or the input function is known only at certain points, such as obtained by sampling. The integral in Eq. (24) is numerically evaluated by means of the Gaussian quadratures using 30-61 quadrature points. A FORTRAN subroutine DQDAG/QDAG (Visual Numerics, Inc., 1997) based on the Gaussian rule, is readily employed to perform the numerical integration. The accuracy of the evaluated results of Eq. (24) using numerical integration is checked by comparing with two specific analytical solutions for exponentially decaying and sinusoidally periodic input functions. Figures 4 and 5 show the results from the numerical integration of Eq. (24) and the two specific analytical solutions for exponentially decaying and sinusoidally periodic input functions, respectively. The applicability of Eq. (24) is illustrated by excellent agreements between the results from numerical integration of Eq. (24) and the specific analytical solutions for both cases (Figs. 5 and 6).

\section{Conclusions}

This study derives a generalized analytical solution for onedimensional advective-dispersive transport in finite spatial domain subject to arbitrary time-dependent inlet boundary condition. The analytical procedures consists of taking Laplace transform with respect to time and generalized integral transform with respect to spatial coordinate. Three simple time-dependent inlet conditions including constant, exponentially decaying and sinusoidally periodic input functions are considered to demonstrate the applicability of the generalized analytical solution for development of the specific analytical solution for some specified input function. Specifically, parametric analysis is performed to illustrate the salient behavior of solute transport resulting from a periodic input function. Moreover, the generalized solution which consists of an integral representation is also evaluated by means of the numerical integration to extend its usage. The generalized analytical solution provides the foundation for deriving analytical solution for some specified types of the time-dependent inlet condition or numerically evaluating the concentration distribution for arbitrary time-dependent inlet boundary condition. Furthermore, the solution derived for sinusoidal periodic function will be added to the compendium

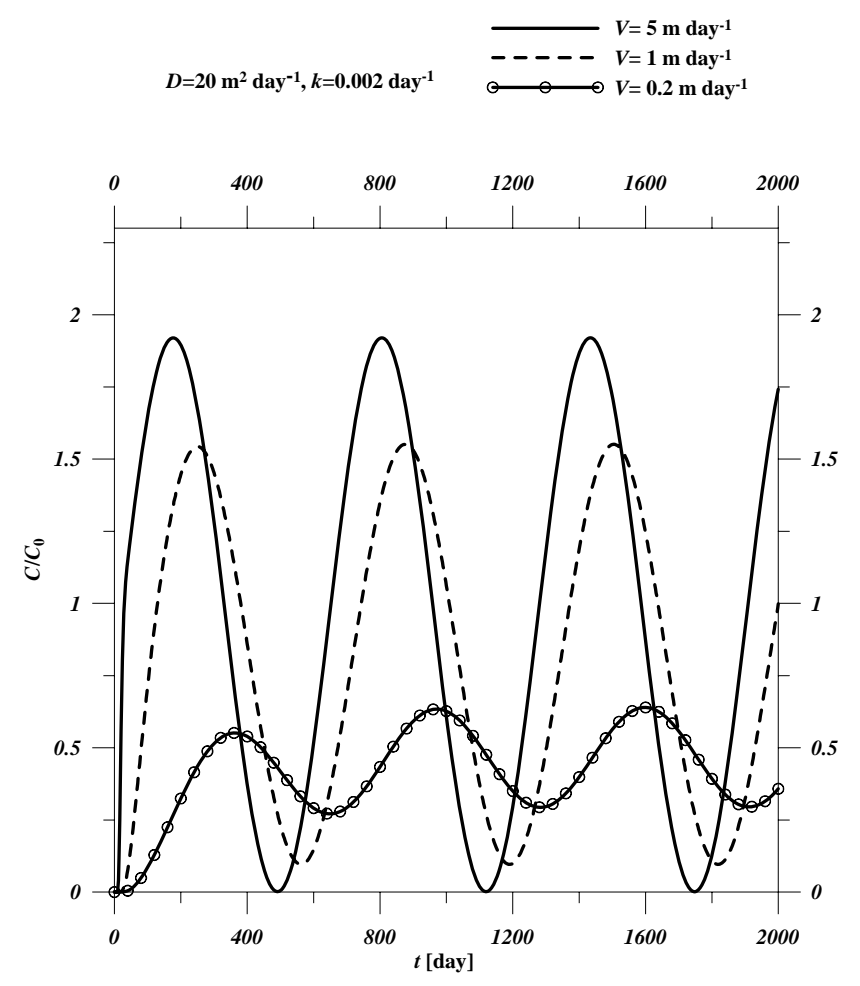

Fig. 4. Comparison of the breakthrough curves at $x=100 \mathrm{~m}$ for different $V$. The sinusoidally periodic input function is $f(t)=1+$ $\sin t$. Parameter $V$ is varied and other parameters are kept constant.

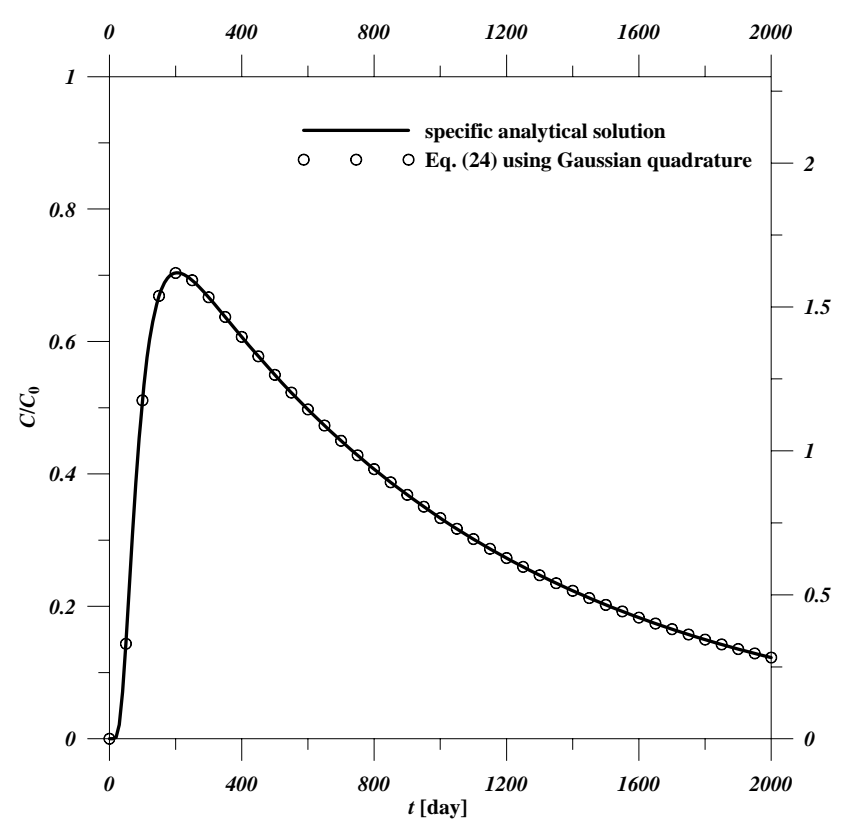

Fig. 5. Comparison of the breakthrough curves at $x=100 \mathrm{~m}$ from the numerical integration of Eq. (24) and the specific analytical solution for exponentially decaying input function $(f(t)=\exp (-t))$. 


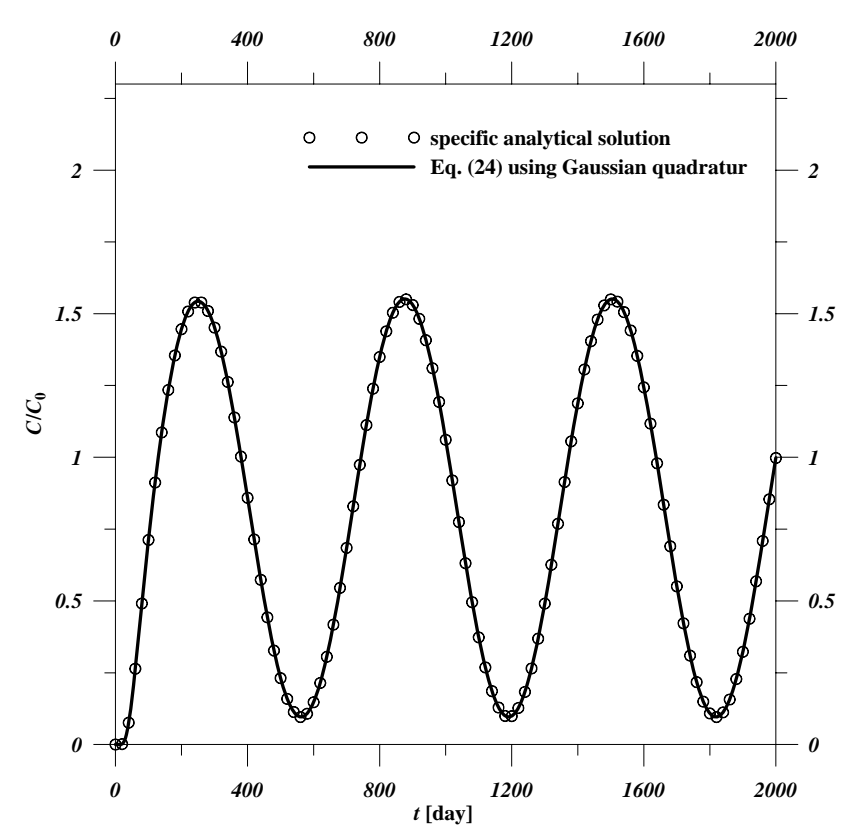

Fig. 6. Comparison of the breakthrough curves at $x=100 \mathrm{~m}$ from the numerical integration of Eq. (24) and the specific analytical solution for sinusoidal periodic input function $(f(t)=1+\sin t)$.

of the analytical solutions to the advection-dispersion equation reported by other researchers in literature. The analytical solution for finite spatial domain associated with timedependent inlet boundary condition should be particularly useful for verification of the more comprehensive numerical models, because some field numerical simulations generally involve finite domain and time-dependent source boundary conditions. We can also conclude that the developed generalized analytical solution serves as a useful tool for the development of the analytical solutions for some specified time-dependent input functions or numerical evaluation of concentration distribution for arbitrary time-dependent input function.

\section{Appendix A}

\section{The derivation of the specific analytical solution}

In this appendix, we present the derivation procedures of the analytical solutions for sinusoidally periodic time-dependent input function $\left(f(t)=C_{0} \sin (\omega t)\right.$ or $f\left(t_{\mathrm{D}}\right)=C_{0} \sin \left(\frac{R \omega L}{V} t_{\mathrm{D}}\right)$ using Eq. (24).
First, inserting $f\left(t_{\mathrm{D}}\right)=C_{0} \sin \left(\frac{R \omega L}{V} t_{\mathrm{D}}\right)$ into the Eq. (24) yields

$$
\begin{aligned}
& C\left(x_{\mathrm{D}}, t_{\mathrm{D}}\right)=C_{0} \sin \left(\frac{R \omega L}{V} t_{\mathrm{D}}\right)-\sum_{m=1}^{\infty} \exp \left(\frac{\mathrm{Pe}}{2} x_{\mathrm{D}}\right) E\left(\psi_{m}, x_{\mathrm{D}}\right) F\left(t_{\mathrm{D}}\right) \\
& F\left(t_{\mathrm{D}}\right)=C_{0} \sin \left(\omega_{\mathrm{D}} t_{\mathrm{D}}\right)-\left(\frac{\psi_{m}^{2}}{\mathrm{Pe}}+\frac{\mathrm{Pe}}{4}\right) e^{-\left(\frac{\psi_{m}^{2}}{\mathrm{Pe}}+\frac{\mathrm{Pe}}{4}+k_{\mathrm{D}}\right) t_{\mathrm{D}}} \\
& \int_{0}^{t_{\mathrm{D}}} C_{0} \sin \left(\omega_{\mathrm{D}} \tau\right) e^{\left(\frac{\psi_{m}^{2}}{\mathrm{Pe}}+\frac{\mathrm{Pe}}{4}+k_{\mathrm{D}}\right) \tau} d \tau
\end{aligned}
$$

The integral representation term in Eq. (A2) can be evaluated using the following integration formula

$$
\int e^{a x} \sin b x=\frac{e^{a x}}{a^{2}+b^{2}}(a \sin b x-b \cos b x)+c
$$

Making use of Eq. (A3) on (A2), Eq. (A2) can be expressed as in dimensional form as

$$
\begin{aligned}
& F\left(t_{\mathrm{D}}\right)=\frac{\omega_{\mathrm{D}}^{2}+\left(\frac{\psi_{m}^{2}}{\mathrm{Pe}}+\frac{\mathrm{Pe}}{4}+k_{\mathrm{D}}\right)^{2}-\left(\frac{\psi_{m}^{2}}{\mathrm{Pe}}+\frac{\mathrm{Pe}}{4}\right)\left(\frac{\psi_{m}^{2}}{\mathrm{Pe}}+\frac{\mathrm{Pe}}{4}+k_{\mathrm{D}}\right)}{\omega_{\mathrm{D}}^{2}+\left(\frac{\psi_{m}^{m}}{\mathrm{Pe}}+\frac{\mathrm{Pe}}{4}+k_{\mathrm{D}}\right)^{2}} \sin \left(\omega_{\mathrm{D}} t_{\mathrm{D}}\right) \\
& +\frac{\left(\frac{\psi_{m}^{2}}{\mathrm{Pe}}+\frac{\mathrm{Pe}}{4}\right) \omega_{\mathrm{D}}}{\omega_{\mathrm{D}}^{2}+\left(\frac{\psi_{m}^{2}}{\mathrm{Pe}}+\frac{\mathrm{Pe}}{4}+k_{\mathrm{D}}\right)^{2}} \cos \left(\omega_{\mathrm{D}} t_{\mathrm{D}}\right)-\frac{\left(\frac{\psi_{m}^{2}}{\mathrm{Pe}}+\frac{\mathrm{Pe}}{4}\right) \omega_{\mathrm{D}}}{\omega_{\mathrm{D}}^{2}+\left(\frac{\psi_{m}^{2}}{\mathrm{Pe}}+\frac{\mathrm{Pe}}{4}+k_{\mathrm{D}}\right)^{2}} e^{-\left(\frac{\psi_{m}^{2}}{\mathrm{Pe}}+\frac{\mathrm{Pe}_{\mathrm{e}}}{4}+k_{\mathrm{D}}\right) t_{\mathrm{D}}}
\end{aligned}
$$

Rearranging the terms and introducing the dimensional variables, Eqs. (A1) and (A2) have the following form

$$
C\left(x_{\mathrm{D}}, t_{\mathrm{D}}\right)=C_{b}\left[G_{1}(x, t)+G_{2}(x, t)-G_{3}(x, t)\right]
$$

where

$$
\begin{aligned}
& G_{1}(x, t)=\left[1-\sum_{m=1}^{\infty} \frac{E\left(\psi_{m}, x\right)\left\{\left[\psi_{m}^{2}+\left(\frac{\mathrm{VL}}{2 D}\right)^{2}+\frac{k L^{2}}{D}\right] \frac{k L^{2}}{D}+\frac{\omega \mathrm{NL}^{2}}{D}\right\} \exp \left(\frac{V x}{2 D}\right)}{\left[\psi_{m}^{2}+\left(\frac{\mathrm{VL}}{2 D}\right)^{2}+\frac{k L^{2}}{D}\right]^{2}+\left(\frac{\omega \mathrm{RL}^{2}}{D}\right)^{2}}\right] \sin (\omega t) \\
& G_{2}(x, t)=\sum_{m=1}^{\infty} \frac{E\left(\psi_{m}, x\right)\left\{\left[\psi_{m}^{2}+\left(\frac{\mathrm{VL}}{2 D}\right)^{2}\right] \frac{\omega \mathrm{RL}^{2}}{D}\right\} \exp \left(\frac{V x}{2 D}\right)}{\left[\psi_{m}^{2}+\left(\frac{\mathrm{VL}}{2 D}\right)^{2}+\frac{k L^{2}}{D}\right]^{2}+\left(\frac{\omega \mathrm{RL}}{D}\right)^{2}} \cos (\omega t) \\
& G_{3}(x, t)=\sum_{m=1}^{\infty} \frac{E\left(\psi_{m}, x\right)\left\{\left[\psi_{m}^{2}+\left(\frac{\mathrm{VL}}{2 D}\right)^{2}\right] \frac{\omega \mathrm{RL}^{2}}{D}\right\} \exp \left(\frac{V x}{2 D}-\frac{k t}{R}-\frac{V^{2} t}{4 D R}-\frac{\beta_{m}^{2} D t}{L^{2} R}\right)}{\left[\psi_{m}^{2}+\left(\frac{\mathrm{VL}}{2 D}\right)^{2}+\frac{k L^{2}}{D}\right]^{2}+\left(\frac{\omega \mathrm{NL}^{2}}{D}\right)^{2}}
\end{aligned}
$$

Acknowledgements. The authors would like to thank the National Science Council of the Republic of China for financially supporting this work under Contract No. NSC 100-3113-E-008-002.

Edited by: A. Gelfan 


\section{References}

Batu, V.: A generalized two-dimensional analytical solution for hydrodynamic dispersion in bounded media with the first-type boundary condition at the source, Water Resour. Res., 25(6), 1125-1132, 1989.

Batu, V.: A generalized two-dimensional analytical solute transport model in bounded media for flux-type finite multiple sources, Water Resour. Res., 29(8), 2881-2892, 1993.

Batu, V.: A generalized three-dimensional analytical solute transport model for multiple rectangular first-type sources, J. Hydrol., 174(1-2), 57-82, 1996.

Chen, J. S., Liu, C. W., Chen, C. S., and Yeh, H. D.: A Laplace transform solution for tracer tests in a radially convergent flow field with upstream dispersion, J. Hydrol., 183, 263-275, 1996.

Chen, J. S., Liu, Y.H., Liang, C. P., Liu, C. W., and Lin, C. W.: Exact analytical solutions for two-dimensional advectiondispersion equation in cylindrical coordinates subject to thirdtype inlet boundary condition, Adv. Water Resour., 34(3), 365374, $2011 \mathrm{a}$.

Chen, J. S., Chen, J. T., Liu, C. W., Liang, C. P., and Lin, C. W.: Analytical solutions to two-dimensional advection-dispersion equation in cylindrical coordinates in finite domain subject to firstand third-type inlet boundary conditions, J. Hydrol., 405(3-4), 522-531, 2011b

Clearly, R. W. and Adrian, D. D.: Analytical solution of the convective-dispersive equation for cation adsorption in soils, Soil Sci. Soc. Am. Proc., 37, 197-199, 1973.

Cotta, R. M. and Milkhailov, M. D.: Heat Conduction: Lumped Analysis, Integral Transforms, Symbolic Computations, WileyInterscience, New York, 1997.

Leij, F. J., Skaggs, T. H., and van Genuchten, M. Th.: Analytical solution for solute transport in three-dimensional semi-infinite porous media, Water Resour. Res., 27(10), 2719-2733, 1991.

Leij, F. J., Toride, N., and van Genuchten, MTh.: Analytical solutions for non-equilibrium solute transport in three-dimensional porous media, J. Hydrol., 151(2-4), 193-228, 1993.

Logan, J. D. and Zlotnik, V.: The convection-diffusion equation with periodic boundary condtion, Appl. Math. Lett. 8(3), 55-61, 1995.

Logan, J. D. and Zlotnik, V.: Time-periodic transport in heterogeneous porous media, Appl. Math. Comput., 75, 119-138, 1996.
Moridis, G. J. and Reddell, D. L.: The Laplace transform finite difference method for simulation of flow through porous media, Water Resour. Res., 27(8), 1873-1884, 1991.

Park, E. and Zhan, H.: Analytical solutions of contaminant transport from finite one-, two, three-dimensional sources in a finitethickness aquifer, J. Contam. Hydrol., 53(1-2), 41-61, 2001.

Pérez Guerrero, J. S., Pimentel, L. C. G., Skaggs, T. H., and van Genuchten, M. Th.: Analytical solution of the advectiondiffusion transport equation using a change-of-variable and integral transform technique, Int. J. Heat. Mass Transf., 52, 3297 3304, 2009a.

Pérez Guerrero, J. S., Pimentel, L. C. G., Skaggs, T. H., and van Genuchten, M. Th.: Analytical solution for multi-species contaminant transport subject to sequential first-order decay reactions in finite media, Transport Porous Med., 80, 373-357, 2009a.

Pérez Guerrero, J. S. and Skaggs, T. H.: Analytical solution for one-dimensional advection-dispersion transport equation with distance-dependent coefficients, J. Hydrol., 390(1-2), 57-65, 2010a.

Pérez Guerrero, J. S, Skaggs, T. H., van Genuchten, M. Th.: Analytical solution for multi-species contaminant transport in finite media with time-varying boundary conditions, Transport Porous Med., 85, 171-178, 2010b.

Ozisik, M. N.: Heat Conduction, Wiley, New York, 1980.

Selim, H. M. and Mansell, R. S.: Analytical solution for transport of reactive solutes through soils, Water Resour. Res., 12(3), 528532, 1976.

van Genuchten, M. Th. and Alves, W. J.: Analytical Solutions of the One-Dimensional Convective-Dispersive Solute Transport Equation. U.S. Department of Agriculture, Technical Bulletin No. 1661, 151 pp., 1982.

Visual Numerics, Inc., IMSL User's Manual. Houston, TX, USA, 1, 591-594, 1997.

Yeh, H. D. and Yeh, G. T.: Yeh, Analysis of point-source and boundary-source solutions of one-dimensional groundwater transport equation, J. Environ. Eng. ASCE, 133(11), 1032-1041, doi:10.1061/(ASCE)0733-9372(2007)133:11(1032), 2007.

Zhan, H., Wen, Z., and Gao, G.: An analytical solution of two-dimensional reactive solute transport in an aquifer-aquitard system, Water Resour. Res., 45, W10501, doi:10.1029/2008WR007479, 2009. 